\title{
TRAVELLING WAVE SOLUTIONS FOR TWO COUPLED NONLINEAR EVOLUTION EQUATIONS BY USING MODIFIED DIRECT ALGEBRAIC METHOD
}

\section{GUIYING CHEN}

College of Mathematics Science

Liaocheng University

Liaocheng 252059

Shandong

P. R. China

e-mail: chengquiying@126.com

\begin{abstract}
Based on a modified direct algebraic method, the Jacobi elliptic function travelling wave solutions for the coupled system of variant Boussinesq equations and the coupled system of KdV equations are obtained. The present solutions can degenerate to the hyperbolic function solutions or the trigonometric function solutions which have been proposed in the previous works if the modulus $m$ in Jacobi elliptic functions satisfies $m \rightarrow 1$ or $m \rightarrow 0$. Moreover, the method can be applied to a wide class of coupled nonlinear evolution equations.
\end{abstract}

2010 Mathematics Subject Classification: 35Q51, 35-04.

Keywords and phrases: modified direct algebraic method, travelling wave solutions, coupled system of variant Boussinesq equations, coupled system of KdV equations.

Supported by National Science Foundation of China Under Grants 11076015.

Received June 8, 2016

(C) 2016 Scientific Advances Publishers 


\section{Introduction}

In recent years, the investigation of nonlinear evolution equations (NLEEs) has played a significant role in many scientific applications, such as electromagnetic, acoustics, electrochemistry, cosmology, and fluid mechanics. Various approaches to searching for the travelling wave solutions of NLEEs have been proposed including the inverse scattering method [1-3], Darboux transformation method [4-6], Bäcklund transformation method [7, 8], Hirota bilinear method [9-11], Lie group analysis method [12, 13], and so on. In recent years, a direct algebraic method is used in [14] for solving the coupled partial differential equations to obtained the hyperbolic function solutions and the trigonometric function solutions. In this article, the direct algebraic method is modified, and by this method, the Jacobi elliptic function travelling solutions are presented for the coupled system of variant Boussinesq equations and coupled system of KdV equations.

The rest of this paper is organized as follows: In Section 2, the modified direct algebraic method is presented. In Section 3, we implement this method for finding the Jacobi elliptic function travelling wave solutions of the coupled system of variant Boussinesq equations and the coupled system of KdV equations. The conclusion is drawn in the last section.

\section{The Modified Direct Algebraic Method}

The following is a given nonlinear coupled partial differential equation with two variables $x$ and $t$ :

$$
F\left(u, v, u_{t}, v_{t}, u_{x}, v_{x}, u_{x x x}, v_{x x x}\right)=0 .
$$

Our method mainly consists three steps:

Step 1. Assume that Equation (1) has the following travelling wave solutions:

$$
u(x, t)=u(\xi)=\sum_{i=1}^{m_{1}} a_{i} G^{i}(\xi), \quad v(x, t)=v(\xi)=\sum_{i=1}^{m_{2}} b_{i} G^{i}(\xi),
$$


and

$$
G^{2}=\alpha_{0}+\alpha_{2} G^{2}+\alpha_{4} G^{4}, \quad \xi=k x+l t
$$

where $a_{i}, b_{i}, \alpha_{i}, k$, and $l$ are arbitrary constants. We know that Equation (3) has Jacobi elliptic function solutions such as $s n, c n, d n$, etc.

Step 2. Balancing the highest order derivative term and the highest order nonlinear term of Equation (1), the coefficients of the series $a_{1}, a_{0}, \ldots, a_{m_{1}}, b_{0}, b_{1}, \ldots, b_{m_{2}}, \alpha_{0}, \alpha_{2}, \alpha_{4}, k$, and $l$ are parameters that can be determined.

Step 3. By substituting Equations (2) and (3) into Equation (1), and collecting the coefficients of $G^{i} G^{\prime}$, and then setting the coefficients to zero, we will obtain a set of algebraic equations. Taking $G$ to be special Jacobi elliptic function, and solving the algebraic equations, the solutions of Equation (1) can be derived.

\section{The Application of the Direct Algebraic Method}

\subsection{The coupled system of variant Boussinesq equations}

The coupled variant Boussinesq system has been derived to describe bi-directional propagation in various contexts-for instance, a model for water waves [15-18], a Toda lattice model with a transverse degree of freedom [19], a two-layered lattice model [20], and a diatomic lattice [21]. The model can be written in the form $[14,17,18,22]$

$$
u_{t}+u u_{x}+v_{x}+\gamma u_{x x t}=0, \quad v_{t}+u v_{x}+v u_{x}+\lambda v_{x x x}=0
$$

where $\gamma$ and $\lambda$ are constants. Consider the travelling wave solutions of Equations (2) and (3); then Equation (4) becomes

$$
l u^{\prime}+k u u^{\prime}+k v^{\prime}+\gamma k^{2} l u^{(3)}=0, \quad l v^{\prime}+k u v^{\prime}+k v u^{\prime}+\lambda k^{3} u^{(3)}=0 .
$$

Balancing the highest order derivative terms and the highest order nonlinear terms in Equation (5), we can find $m_{1}=2, m_{2}=2$. Suppose the solutions of Equation (4) are of the form 


$$
u(\xi)=a_{0}+a_{1} G+a_{2} G^{2}, \quad v(\xi)=b_{0}+b_{1}+b_{2} G^{2} .
$$

Substituting Equations (6) and (3) into Equation (5) yields a set of algebraic equations for $a_{0}, a_{1}, a_{2}, b_{0}, b_{1}, b_{2}, \alpha_{0}, \alpha_{2}, \alpha_{4}, k$, and $l$. These equations are found as

$$
\begin{gathered}
l a_{1}+k a_{0} a_{1}+k b_{1}+\gamma k^{2} l a_{1} \alpha_{2}=0, \quad 3 k a_{1} a_{2}+6 \gamma k^{2} l a_{1} \alpha_{4}=0, \\
2 l a_{2}+k\left(2 a_{0} a_{2}+a_{1}^{2}\right)+2 k b_{2}+8 \gamma k^{2} l a_{2} \alpha_{2}=0, \quad k a_{2} b_{2}+6 \lambda k^{3} a_{2} \alpha_{4}=0 \\
k a_{2}^{2}+12 \gamma k^{2} l a_{2}^{2} \alpha^{4}=0, \quad l b_{1}+k\left(a_{0} b_{1}+a_{1} b_{0}\right)+\lambda k^{3} a_{1} \alpha_{2}=0, \\
2 l b_{2}+2 k\left(a_{0} b_{2}+a_{1} b_{1}+a_{2} b_{0}\right)+8 \lambda k^{3} a_{2} \alpha_{2}=0 \\
3 k\left(a_{1} b_{2}+a_{2} b_{1}\right)+6 \lambda k^{3} a_{1} \alpha_{4}=0 .
\end{gathered}
$$

Now, we search for the elliptic function solutions of Equation (4).

Case 1. If $\alpha_{0}=m^{2}-1, \alpha_{2}=2-m^{2}$, and $\alpha_{4}=-1$, then $G=d n(k x+l t)$. Solving Equation (7), we obtain the travelling wave solutions of Equation (4):

$$
\begin{gathered}
u(x, t)=\frac{8 k^{2} m^{2} l^{2} \gamma^{2}-16 k^{2} l^{2} \gamma^{2}-k^{2} \lambda-2 l^{2} \gamma}{2 k l \gamma}+12 k l \gamma d n^{2}(k x+l t), \\
v(x, t)=\frac{k^{2} \lambda\left(8 m^{2} l^{2} \gamma^{2}-16 l^{2} \gamma^{2}+\lambda\right)}{4 l^{2} \gamma^{2}}+6 k^{2} \lambda d n^{2}(k x+l t) .
\end{gathered}
$$

Case 2. If $\alpha_{0}=m^{2}\left(m^{2}-1\right), \alpha_{2}=2 m^{2}-1$, and $\alpha_{4}=1$, then $G=d s(k x+l t)$. Solving Equation (7), we obtain the travelling wave solutions of Equation (4):

$$
\begin{gathered}
u(x, t)=-\frac{16 m^{2} k^{2} l^{2} \gamma^{2}-8 k^{2} l^{2} \gamma^{2}+k^{2} \lambda+2 l^{2} \gamma}{2 k l \lambda}-12 k l \gamma d s^{2}(k x+l t), \\
v(x, t)=-\frac{k^{2} \lambda\left(16 m^{2} l^{2} \gamma^{2}-8 l^{2} \gamma^{2}-\lambda\right)}{4 l^{2} \gamma^{2}}-6 k^{2} \lambda d s^{2}(k x+l t) .
\end{gathered}
$$


Remark. Noticed that $d n(\xi) \rightarrow \operatorname{sech}(\xi), d s(\xi) \rightarrow \operatorname{csch}(\xi)$ when the modulus $m \rightarrow 1$, and $d n(\xi) \rightarrow \tan (\xi), d s(\xi) \rightarrow \csc (\xi)$ when $m \rightarrow 0$, we can obtain the corresponding hyperbolic function solutions and triangular-like function solutions of Equation (4) which have been proposed in [14].

Figure 1(a) and (b) represent the dark and bright solitary solutions (8) of the coupled system of variant Boussinesq equations (4) with $k=0.5, l=0.1, m=0.5$ in the interval $x \in[-10,10]$ and $t \in[0,1]$.
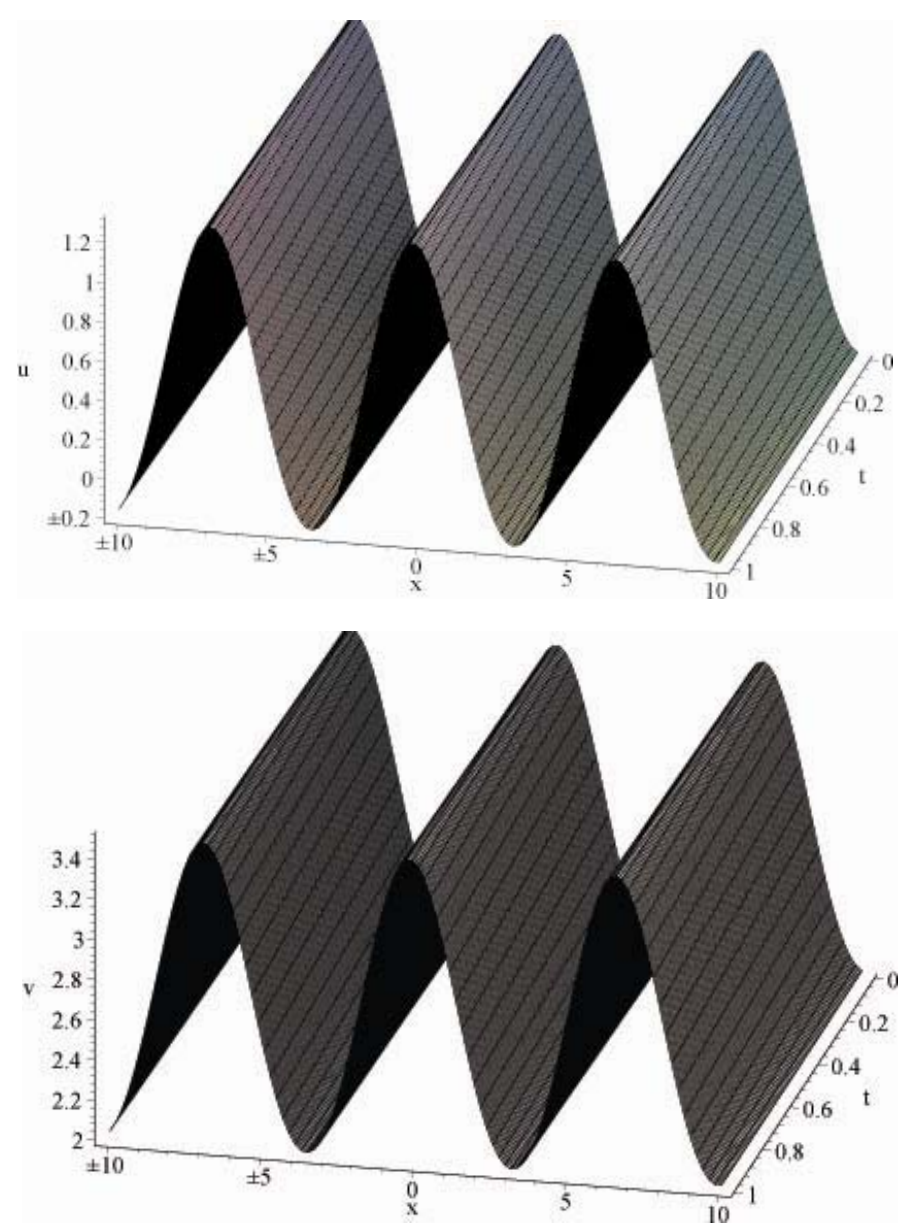

Figure 1. Travelling wave solutions (8) of the variant Boussinesq equations (4). 


\subsection{The coupled system of $\mathrm{KdV}$ equations}

Gear and Grimshaw derived a system of coupled KdV equations to model interactions of long waves, for example, in a stratified fluid. Specially, their model is [14, 23]

$$
u_{t}+\left(u_{x x}+u^{2}+\frac{\varepsilon}{2} v^{2}+\varepsilon u v\right)_{x}=0, \quad v_{t}+\left(v_{x x}+v^{2}+\frac{\varepsilon}{2} u^{2}+\varepsilon u v\right)_{x}=0,
$$

where $\varepsilon$ is constant. Consider the travelling wave solutions of Equation (2). Then Equation (10) becomes

$$
\begin{aligned}
& l u^{\prime}+k^{3} u^{(3)}+2 k u u^{\prime}+k \varepsilon\left(v v^{\prime}+u^{\prime} v+u v^{\prime}\right)=0 \\
& l v^{\prime}+k^{3} v^{(3)}+2 k v v^{\prime}+\varepsilon k\left(u u^{\prime}+u^{\prime} v+u v^{\prime}\right)=0
\end{aligned}
$$

Balancing the highest order derivative terms and the highest order nonlinear terms in Equation (11), we can find $m_{1}=2=m_{2}$. Suppose the solutions of Equation (11) are of the form

$$
u(\xi)=a_{0}+a_{1} G+a_{2} G^{2}, \quad v(\xi)=b_{0}+b_{1}+b_{2} G^{2} .
$$

Substituting Equations (12) and (3) into Equation (11) yields a set of algebraic equations for $a_{0}, a_{1}, a_{2}, b_{0}, b_{1}, b_{2}, \alpha_{0}, \alpha_{2}, \alpha_{4}, k$, and $l$. These equations are found as:

$$
\begin{gathered}
l a_{1}+k^{3} a_{1} \alpha_{2}+2 k a_{0} a_{1}+k \varepsilon\left(b_{0} b_{1}+a_{0} b_{1}+a_{1} b_{0}\right)=0, \\
2 k^{3} b_{1} \alpha_{4}+2 k b_{1} b_{2}+k \varepsilon\left(a_{1} a_{2}+a_{1} b_{2}+a_{2} b_{1}\right)=0, \\
2 a_{2} l+8 k^{3} a_{2} \alpha_{2}+2 k\left(2 a_{0} a_{2}+a_{1}^{2}\right)+k \varepsilon\left(2 b_{0} b_{2}+b_{1}^{2}+2 a_{1} b_{1}+2 a_{2} b_{0}+2 a_{0} b_{2}\right)=0, \\
2 k^{3} a_{1} \alpha_{4}+2 k a_{1} a_{2}+k \varepsilon\left(b_{1} b_{2}+a_{2} b_{1}+a_{1} b_{2}\right)=0, \\
12 k^{3} a_{2} \alpha_{4}+2 k a_{2}^{2}+k \varepsilon\left(b_{2}^{2}+2 a_{2} b_{2}\right)=0,
\end{gathered}
$$




$$
\begin{gathered}
l b_{1}+k^{3} b_{1} \alpha_{2}+2 k b_{0} b_{1}+\varepsilon k\left(a_{0} a_{1}+a_{1} b_{0}+a_{0} b_{1}\right)=0, \\
12 k^{3} b_{2} \alpha_{4}+2 k b_{2}^{2}+k \varepsilon\left(a_{2}^{2}+2 a_{2} b_{2}\right)=0, \\
2 l b_{2}+8 k^{3} b_{2} \alpha_{2}+2 k\left(2 b_{2}^{2}+b_{1}^{2}\right)+k \varepsilon\left(2 a_{0} a_{2}+2 a_{1} b_{1}+2 a_{2} b_{0}+2 a_{0} b_{2}+a_{1}^{2}\right)=0 .
\end{gathered}
$$

Now, we search for the elliptic solutions of Equation (10).

Case 1. If $\alpha_{0}=m^{2}-1, \alpha_{2}=2-m^{2}$, and $\alpha_{4}=-1$, then $G=d n(k x+l t)$. Solving Equation (13), the travelling wave solutions of Equation (10) are found as:

$$
\varepsilon=1, \quad u(x, t)=v(x, t)=-\frac{4 k^{3} m^{2}-8 k^{3}-l}{k}-12 k^{2} d n^{2}(k x+l t),
$$

and

$$
\varepsilon=2, \quad u(x, t)=v(x, t)=-\frac{4 k^{3} m^{2}-8 k^{3}-l}{4 k}-3 k^{2} d n^{2}(k x+l t) .
$$

Case 2. If $\alpha_{0}=1, \alpha_{2}=-\left(m^{2}+1\right)$, and $\alpha_{4}=m^{2}$, then $G=s n(k x+l t)$. The travelling wave solutions of Equation (10) are found as:

$$
\begin{aligned}
\varepsilon=\frac{10}{9} & \\
u(x, t)=v(x, t)=-\frac{9}{8} \frac{k^{3} m^{2}+k^{3}-l}{k} & \pm \frac{9}{4} m \sqrt{2 k^{4} m^{2}+2 k^{4}-5 k l} s n(k x+l t) \\
& -\frac{9}{4} k^{2} m^{2} s n^{2}(k x+l t)
\end{aligned}
$$

and

$$
\varepsilon=4, \quad u(x, t)=v(x, t)=\frac{4 k^{3} m^{2}+4 k^{3}-l}{2 k}-6 k^{2} m^{2} s n^{2}(k x+l t) .
$$


Case 3. If $\alpha_{0}=1-m^{2}, \alpha_{2}=-1+2 m^{2}$, and $\alpha_{4}=-m^{2}$, then $G=c n(k x+l t)$. The travelling wave solutions of Equation (10) are found as:

$$
\begin{aligned}
\varepsilon=\frac{10}{9} & \\
u(x, t)=v(x, t)=-\frac{9}{8} \frac{2 k^{3} m^{2}-k^{3}+l}{k} & \pm \frac{9}{4} m \sqrt{4 k^{4} m^{2}-2 k^{4}+5 k l c n}(k x+l t) \\
& +\frac{9}{4} k^{2} m^{2} c n^{2}(k x+l t)
\end{aligned}
$$

and

$$
\begin{gathered}
\varepsilon=4, \quad u(x, t)=-\frac{8 k^{3} m^{2}-4 k^{3}+l}{2 k}+6 k^{2} m^{2} c n^{2}(k x+l t), \\
v(x, t)=-\frac{8 k^{3} m^{2}+4 k^{3}-l}{8}-12 k^{2} m^{2} c n^{2}(k x+l t) .
\end{gathered}
$$

\section{Discussion and Conclusion}

The direct algebraic method has been modified, and by this method the Jacobi elliptic function on travelling wave solutions for the coupled system of variant Boussinesq equations and the coupled system of $\mathrm{KdV}$ equations are obtained. The solutions are degenerated to hyperbolic function solutions or triangular-like function solutions in the limit cases when the modulus of the Jacobi elliptic functions satisfy $m \rightarrow 1$ or $m \rightarrow 0$, which shows that the method is much simple and powerful to find new solutions to other nonlinear coupled evolution equations in mathematical physics.

\section{References}

[1] M. J. Ablowitz and H. Segur, Solitons and the Inverse Scattering Transform, Philadephia: SIAM, 1981.

[2] S. P. Novikov, S. V. Manakov and L. P. Pitaevskii, Theory of Solitons: The Inverse Scattering Methods, New York: Consultants Bureau, 1984. 
[3] M. J. Ablowitz and P. A. Clarkson, Solitons, Nonlinear Evolution Equations and Inverse Scattering, Cambridge: Cambridge University Press, 1991.

[4] V. B. Matveev and M. A. Salle, Darboux Transformations and Solitons, Berlin: Springer, 1991.

[5] C. H. Gu and Z. X. Zhou, On Darboux transformations for soliton equations in highdimensional spacetime, Lett. Math. Phys. 32(1) (1994), 1-10.

[6] H. C. Hu and Q. P. Liu, New Darboux transformation for Hirota-Satsuma coupled KdV system, Chaos, Solitons and Fractals 17(5) (2003), 921-928.

[7] A. N. W. Hone, V. B. Kuznetsov and O. Ragnisco, Bäcklund transformations for many-body systems related to KdV, J. Phys. A 32 (1999), L299-L306.

[8] D. S. Wang and H. Q. Zhang, Auto Bäcklund transformation and new exact solutions of the (2+1)-dimensional Nizhnik-Novikov-Veselov equation, Int. J. Mod. Phys. C 103(16) (2005), 393-412.

[9] R. Hirota, Exact solution of the Korteweg-de Vries equation for multiple collisions of solitons, Phys. Rev. Lett. 27(18) (1971), 1192.

[10] A. M. Wazwaz, The Hirota's bilinear method and the tanh-coth method for multiplesoliton solutions of the Sawada-Kotera-Kadomtsev-Petviashvili equation, Appl. Math. Comput. 200(1) (2008), 160-166.

[11] H. C. Zhang, W. X. Ma and X. Gu, Hirota bilinear equations with linear subspaces of hyperbolic and trigonometric function solutions, Appl. Math. Comput. 220 (2013), 226-234.

[12] G. W. Bluman and J. D. Cole, Similarity Methods for Differential Equations, Berlin: Springer, 1974.

[13] M. Y. Guo, X. Q. Liu and J. Gao, Differential invariants and group classification of KdV-Burgers equation, Chinese Journal of Quantum Electronics 26(6) (2009), 138-147.

[14] A. R. Seadwawy and K. EI-Rashidy, Traveling wave solutions for coupled nonlinear evolution equations, Math. Comput. Model. (57) (2013), 1371-1379.

[15] M. L. Wang, Solition wave solutions for variant Boussinesq equations, Phys. Lett. A 199(3-4) (1995), 169-172.

[16] E. Fan and Y. Hon, Applications of extended tanh method to special types of nonlinear equations, Appl. Math. Comput. 141(2-3) (2003), 351-358.

[17] Z. Zhang, Q. Bi and J. Wen, Bifurcations of traveling wave solutions for two coupled variant Boussinesq equations in shallow water waves, Chaos Solitons Tractals. 24 (2005), 631-643.

[18] X. L. Yang and J. S. Tang, Extended Fans algebra method and its application to KdV and variant Boussinesq equations, Commu. Theor. Phys. 48 (2007), 1-6.

[19] P. L. Christiansen, P. S. Lomdahl and V. Muto, Nonlinearity 4 (1991), 477. 
[20] K. P. Khusnutdinova, A. M. Samsonov and A. S. Zakharovv, Phys. Rev. E 79 (2009), 656.

[21] J. A. Wattis, Phys. Lett. A 284 (2001), 16.

[22] L. A. Hassanien, R. A. Zait and E. A. Abdel-Salam, Multicnoidal and multitraveling wave solutions for some nonlinear equations of mathematical physics, Phys. Scr. 67 (2003), 457-463.

[23] J. D. Wright and A. Scheel, Solitary waves and their linear stability in weakly coupled KdV equations, Z. Angew. Math. Phys. 58 (2007), 535-570. 